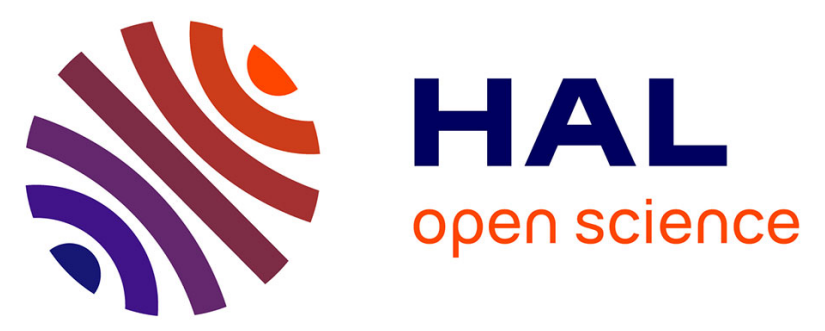

\title{
Combiner les scénarios linguistique et actionnel au sein d'un parcours en ligne d'apprentissage de l'anglais
}

Thomas Gaillat, Martine Schuwer, Sophie Belan

\section{To cite this version:}

Thomas Gaillat, Martine Schuwer, Sophie Belan. Combiner les scénarios linguistique et actionnel au sein d'un parcours en ligne d'apprentissage de l'anglais. Recherche et Pratiques Pédagogiques en Langues de Spécialité: Cahiers de l'APLIUT, 2014, pp.107-120. hal-01171271

\section{HAL Id: hal-01171271 \\ https://hal.science/hal-01171271}

Submitted on 13 Mar 2017

HAL is a multi-disciplinary open access archive for the deposit and dissemination of scientific research documents, whether they are published or not. The documents may come from teaching and research institutions in France or abroad, or from public or private research centers.
L'archive ouverte pluridisciplinaire HAL, est destinée au dépôt et à la diffusion de documents scientifiques de niveau recherche, publiés ou non, émanant des établissements d'enseignement et de recherche français ou étrangers, des laboratoires publics ou privés. 


\title{
Combiner les scénarios linguistique et actionnel au sein d'un parcours en ligne d'apprentissage de l'anglais
}

Thomas Gaillat

Martine Schuwer

Sophie Belan

\section{Résumé}

Cet article aborde la problématique de la construction d'un didacticiel d'apprentissage de l'anglais dans le cadre de la mise en place d'un programme d'auto-formation guidée conjuguant travail à distance et présentiel. L'objectif est de proposer une approche novatrice dans la conception d'un scénario d'apprentissage, à savoir la nécessité de combiner un déroulement pédagogique dans lequel l'apprenant puise le sens de son travail, et un déroulement linguistique assurant l'assimilation de compétences linguistiques. L'approche défend l'idée que le processus d'élaboration d'un parcours résulte de la confrontation d'une démarche actionnelle et d'une pratique raisonnée de la langue.

Mots clé : grammaire, didacticiel en anglais, démarche actionnelle, progression linguistique, acquisition d'une langue étrangère, scénario d'apprentissage, pratique raisonnée de la langue, PRL

\section{Combining linguistic and action-based scenarios in an online English course}

\begin{abstract}
This article focuses on how to design an online course dedicated to learning English as part of a hybrid setup involving online work and student interviews. The objective is to offer a new a approach to the design of a learning scenario, which implies the combination of a pedagogical process, in which the learner finds meaning in his/her work, and of a linguistic process which ensures his/her assimilation of linguistic skills. In our approach, we claim that the process of designing a course is the result of the confrontation of a task-based process and a process which makes learners think about language.
\end{abstract}

Keywords: grammar, online English course, task-based learning, linguistic progression, second language acquisition, SLA, teaching scenario, language conceptualisation approach, e-education didactics 


\section{Introduction}

La présente étude rend compte de l'expérience et des dispositifs mis en place au sein du Master professionnel «Linguistique et Didactique des Langues » à l'Université Rennes 2, dans le cadre d'un projet de création de didacticiels d'anglais pour le programme d'auto-formation guidée d'étudiants du secteur LANSAD de l’Université de Rennes 1.

Dans cette université, au début des années 2000, des dispositifs d'auto-formation guidée (AFG) en langues ont été mis en place par l'équipe du SCELVA (Service commun d'étude des langues vivantes appliquées) pour répondre à des contraintes institutionnelles (faibles volumes horaires en langues dans certaines filières) et organisationnelles (effectifs nombreux et ressources humaines limitées). L'objectif de ces dispositifs était d'optimiser le temps d'enseignement en langues en articulant temps de travail en présentiel et activités à réaliser à distance. L'introduction, en 2005, de la plate-forme Moodle a permis aux concepteurs de développer davantage ces dispositifs et d'offrir aux étudiants l'accès à des ressources plus variées.

L'expérience présentée dans cet article, menée à partir de 2007 et issue du partenariat entre les deux universités rennaises, poursuivait un double objectif. Il s'agissait premièrement de donner aux étudiants de Master 2 Linguistique et Didactique des Langues - Anglais Langue Étrangère - la possibilité de mettre en pratique leurs connaissances théoriques et leurs compétences dans le domaine des TICE par la création de didacticiels d'anglais. Deuxièmement, les étudiants de Licence 2 Sciences bénéficiaient de ces modules dans le cadre de leur parcours d'auto-formation guidée. Ce parcours incluait du travail à distance grâce aux modules qui donnaient suite à des mises en situation par entretiens en trinômes avec un lecteur de langue. Dans chaque module, un intérêt particulier était porté aux thématiques liées aux spécialités des étudiants, mais l'accent était également mis sur les aspects linguistiques et sur la composante actionnelle de l'apprentissage. Ces modules didacticiels sont l'objet de cet article qui vise à présenter comment une approche linguistique de la grammaire s'articule avec la composante actionnelle dans le cadre de l'AFG. L'originalité de l'approche réside dans le fait que l'élaboration dépasse la simple déclinaison d'exercices de grammaire sur un thème donné. C'est en fonction d'un scénario actionnel que les contenus linguistiques du didacticiel sont médiatisés afin de dépasser l'incompatibilité apparente des approches actionnelle et grammaticale

Pour l'auteur du didacticiel, l'objectif est de faire travailler l'apprenant pour une acquisition de type linguistique, de manière sous-jacente et par conséquent de mettre en place un continuum d'activités qui, dans leur progression, doivent faire sens pour l'apprenant : c'est dans l'articulation même de ces activités que réside l'efficacité du parcours d'apprentissage. Il s'agit ainsi, en premier lieu, d'organiser les activités autour de la réalisation de tâches réalistes qui constituent un objectif pragmatique pour l'apprenant. Puis, une fois le parcours actionnel constitué (et en fonction de celui-ci), de définir les activités de type proprement linguistique au sens où les apprenants réfléchissent au fonctionnement de la langue du point de vue fonctionnel plutôt que normatif (Frei 2011, 25).

Un ensemble d'exigences, voire de difficultés, parsèment le chemin de l'élaboration. Une fois construit le scénario actionnel, les contenus sont médiatisés en fonction de plusieurs exigences :

- la richesse informationnelle doit être en phase avec le déroulement logique des actions ;

- le choix d'un ou deux points linguistiques est lié à leur présence effective dans tous les documents du parcours ;

- les « affordances ${ }^{1} »$ des ressources créées doivent correspondre à l'usage prévu en ligne.

$1 \quad$ Selon Uyttebrouk (2005 : 44) le terme « désigne les propriétés fondamentales d’un objet (réel ou virtuel) qui conditionnent et délimitent ses usages potentiels ». 
Le tout constitue ainsi un ensemble homogène d'activités cohérentes du point de vue de leur déroulement.

Dans la première section de cet article, on montrera donc dans quelle mesure il est possible de dépasser l'antagonisme mentionné supra entre l'approche actionnelle et l'approche grammaticale, et on s'interrogera sur la procédure grammaticale à déployer dans la conception d'un parcours en ligne d'apprentissage de l'anglais. La deuxième section présente les fondements théoriques de cette approche. La troisième traite de l'ordre didactique des activités et la quatrième propose l'ergonomie type d'un parcours.

\section{Cadre théorique}

Nos choix méthodologiques s'inscrivent dans deux approches l'approche actionnelle et la pratique raisonnée de la langue. La première section analyse les différences entre ces deux approches et la deuxième section montre dans quelle mesure elles peuvent être compatibles.

\subsection{L'approche actionnelle et la PRL}

Le cadre posé par le Cadre européen de référence pour les langues (CECRL) s’inscrit dans celui de la démarche actionnelle. Le CECRL souligne la nécessité de proposer à l'apprenant une démarche fondée sur des actions dont la réalisation est observable, notamment d'un point de vue langagier : " Le progrès dans l'apprentissage d'une langue apparaît le mieux dans la capacité de l'apprenant à s'engager dans une activité langagière observable " (Conseil de l'Europe 2001: 48). La notion d'engagement place l'apprenant en position d'acteur dans une situation de communication, acteur qui prend part aux activités et produit un résultat observable à travers la langue. La situation constitue le cadre dans lequel se déroulent les actions et assure leur cohérence. Les auteurs du CECRL insistent sur la perspective de type actionnel « en ce qu'elle considère avant tout l'usager et l'apprenant d'une langue comme des acteurs sociaux ayant à accomplir des tâches (qui ne sont pas seulement langagières) dans des circonstances et un environnement donnés, à l'intérieur d'un domaine d'action particulier » (idem : 15). En d'autres termes, il s'agit de proposer des tâches qui ne sont pas des exercices de langue en tant que tels mais des activités menant à l'accomplissement d'un projet ou d'une mission. Une tâche n'est, de toute façon, jamais un exercice de langue (Ellis 2003).

Le cadre théorique de la construction de notre parcours à distance est aussi celui défini dans l'approche énonciativiste de la pratique raisonnée de la langue (PRL). L’arrêté du 14 mars 1986 des Instructions Officielles sur les dispositions communes à l'ensemble des langues vivantes au lycée consacre l'importance de cette dimension : «Pour l'ensemble des langues dont l'étude a été commencée au collège, les programmes des lycées offrent des orientations communes à l'ensemble des séries : [...] Réflexion progressivement affinée sur le fonctionnement de la langue et sur le langage » (Ministère de l'Éducation Nationale 1998 : 15). Le parcours d'apprentissage d'une langue comprendrait donc une part consacrée à l'analyse par l'apprenant de faits de langues, et de ce que ceux-ci révèlent du point de vue " des concepts sous-jacents à l'activité de langage » (Ministère de l'Éducation Nationale, 1998 : 22). Ainsi, au delà de ses spécificités morphosyntaxiques et phonologiques, la langue est vue et enseignée pour que l'apprenant comprenne les opérations qui régissent son fonctionnement. La mise en place d'activités d'observation, d'analyse, de description, de classement, de comparaison est suggérée de manière à favoriser cette conceptualisation.

Mais il s'agit là des Instructions Officielles (IO) qui précédaient l'avènement du CECRL. Les descripteurs ont, depuis, réduit le champ de cette vision conceptualisante de l'apprentissage de la langue. En effet, les IO des années précédant le CECRL octroyaient une place importante à la PRL. Celle-ci a ensuite diminué avec le CECRL qui, à son tour, a ramené la grammaire à une fonction plus descriptive : 
La plupart des linguistes descriptifs se contentent désormais de codifier la pratique, mettant en rapport forme et sens et utilisant une terminologie qui ne s'éloigne de la pratique traditionnelle que lorsqu'il faut traiter des phénomènes extérieurs à la gamme des modèles de description traditionnels. C’est l'approche adoptée dans la Section 5.2. Elle s'efforce d'identifier et de classer les composantes principales de la compétence linguistique définie comme la connaissance des ressources formelles à partir desquelles des messages corrects et significatifs peuvent être élaborés et formulés et la capacité à les utiliser. (CECRL , 2000 : 87)

En d'autres termes, les auteurs laissent à l'enseignant le soin de l'explication du fonctionnement de la langue et abordent la question grammaticale du point de vue descriptif.

[...] toute langue a une grammaire extrêmement complexe qui ne saurait, à ce jour, faire l'objet d'un traitement exhaustif et définitif. Un certain nombre de théories et de modèles concurrents pour l'organisation des mots en phrases existent. Il n'appartient pas au Cadre de référence de porter un jugement ni de promouvoir l'usage de l'un en particulier. Il lui revient, en revanche, d'encourager les utilisateurs à déclarer leur choix et ses conséquences sur leur pratique. Nous nous contentons ici, d'identifier des paramètres et des catégories largement utilisés pour la description grammaticale. (CECRL 2001 : 89)

\subsection{La recherche d'une synthèse}

En fin de compte, on peut dire que la publication du CECRL a opéré un déplacement de focus dans les pratiques d'enseignement en conférant à la situation et à l'action le rôle de catalyseur de l'activité langagière. Il s'en est suivi une diminution de la place octroyée à l'approche grammaticale et de sa mise en œuvre raisonnée. La langue est désormais considérée comme outil dans l'action. L'action guide l'usage de la langue, et la grammaire est utile pour ce qu'elle permet du point de vue fonctionnel. Pour les auteurs du Cadre Européen, l'approche linguistique « s'efforce d'identifier et de classer les composantes principales de la compétence linguistique définie comme la connaissance des ressources formelles à partir desquelles des messages corrects et significatifs peuvent être élaborés et formulés et la capacité à les utiliser » (Conseil de l'Europe 2001 : 86). Ils laissent aux professionnels de la didactique le choix de la manière d'enseigner les concepts relatifs au fonctionnement de la langue en les orientant toutefois vers une approche socio-linguistique de la pratique. Selon Danielle Chini, «s'il est vrai que jusqu"ici la dimension sociolinguistique était injustement laissée pour compte, il ne faudrait pas que sa valorisation entraîne un renversement de balancier au détriment de la dimension cognitive et psycholinguistique fondamentale de l'apprentissage » (2009: 142). L'auteur insiste sur l'étape d'apprentissage que constitue la conceptualisation. Les concepts énonciatifs doivent être introduits dans le processus d'apprentissage pour mener le sujet à concevoir la langue du point de vue des opérations qui la sous-tendent et, ainsi, « dépasser l'irréductibilité des spécificités morphosyntaxiques de surface pour accéder à une perception des enjeux langagiers » (Chini, 2009 : 143).

Barbazan (2011 Section 2.1) rejoint Chini en rappelant l'objectif de la grammaire : rendre compte du fonctionnement de la langue. Ce n'est pas en limitant l'enseignement à une approche descriptive que le fonctionnement interne de la langue est étudié. Or la compréhension de ce fonctionnement interne est nécessaire au transfert des structures grammaticales dans différents contextes. De même,

[l]'organisation morpho-syntaxique de la phrase est mise en relation avec l'organisation du texte, les mécanismes de sa cohérence et de son intelligibilité, et au-delà, avec le type de discours, la situation d'énonciation, sa dimension pragmatique. (Bulletin Officiel, $2010: 6$ )

Comme on le voit, la PRL est une démarche qui se fonde sur l'analyse des faits de langue en contexte. Le contexte permet en effet plusieurs expositions complémentaires au même fait de langue et, par conséquent, favorise la compréhension profonde des opérations régissant le fonctionnement discursif. 
Ces deux "cadres » (celui induit par le CECRL et celui de la PRL) semblent entretenir un rapport dialectique, et la question de leur compatibilité se pose lors de l'élaboration d'un parcours en ligne d'apprentissage d'une langue. Nous pensons qu'il peut y avoir une synthèse des deux pour proposer un apprentissage de la langue intégrant à la fois dynamisme de l'action et prise de recul sur l'objet langue pour permettre la réflexion et favoriser l'apprentissage. L'élaboration d'un parcours en langue se trouve confrontée à cette synergie, et la réflexion sur l'ordre didactique des activités permet de transcender ce rapport dialectique, et ainsi de concilier approche actionnelle et PRL.

\section{Ordre didactique dans le scénario d'apprentissage}

La question de la conciliation des deux approches trouve une réponse chez Chini (2009). Il s'agit de considérer l'apprenant non seulement comme sujet social et sujet énonciateur, mais aussi comme sujet apprenant chargé d'acquérir les outils opératifs propres à la construction et à l'interprétation du discours. Par conséquent, l'ordre didactique des activités d'un parcours prend en compte cette dualité. Reste à savoir dans quel ordre doivent apparaître les activités. Portine apporte un éclairage essentiel en observant « qu'un apprenant doit à la fois structurer correctement ses énoncés (correction ou " accuracy ») et se préparer à agir en temps réel (habileté langagière ou "fluency »), mais il ne peut faire les deux en même temps ; thématiquement, il faut donc subordonner l'un à l'autre » (2009 : 31). Partant de ce constat, il s'agit d'appliquer le principe de subordination en considérant que l'apprenant doit tout d'abord être motivé par le dynamisme de l'action qui donne du sens à sa progression dans le parcours. L'habilité langagière précède alors la correction. Car c'est au sein du déroulement de l'action, et donc des activités d'apprentissage qui la sous-tendent, que la pratique raisonnée de la langue peut se développer.

Pour l'apprenant, le scénario est double. D'une part, il accomplit des tâches le menant vers la réalisation de son projet. D'autre part, il suit une progression linguistique qui s'insère entre les activités actionnelles, voire en leur sein. Par exemple, lorsqu'un étudiant de Licence 2 Sciences se voit proposer une tâche de compréhension d'une séquence vidéo expliquant la construction d'un télescope, les activités de type actionnel se concentrent sur les informations utiles à la réalisation du projet, tandis que les activités linguistiques portent sur un ou plusieurs énoncés présents dans le même contexte. La production langagière est liée au contexte, ce qui doit permettre la compréhension des choix faits par l'énonciateur.

La progression dans le parcours par l'apprenant se fait par le biais d'une interface qui doit véhiculer à la fois le projet et le travail linguistique. Les ressources authentiques en sont le point d'entrée commun. Elles constituent l'interface naturelle entre l'apprenant d'un côté, les tâches et points linguistiques de l'autre. Les ressources subordonnent les tâches, elles-mêmes subordonnant les points linguistiques. Le déroulement de l'apprentissage au sein du parcours se fait donc par l'exposition à des ressources authentiques, enrichie par des activités sur les contenus informationnels, puis sur des points linguistiques du même contexte. Le scénario menant à l'accomplissement du projet inclut nécessairement plusieurs ressources, ce qui multiplie le nombre de tâches de type actionnel et d'activités de type linguistique. Pour chaque ressource, sont imbriquées des actions et des activités sur des faits de langue. Cette imbrication des ressources n'est possible que grâce à la mise en ligne car la plate-forme permet de choisir l'ordre d'apparition des activités qui se fait en fonction de l'évolution de l'apprenant dans le scénario actionnel. Des feedbacks informationnels et linguistiques jalonnent la progression, ce qui la rend interactive. Grâce à la plate-forme, les feedbacks sont spécifiques aux activités et aux événements (erreurs ou questions de compréhension). Par exemple, un glossaire d'erreurs grammaticales est lié aux entrées d'un forum d'expression et les segments erronés sont automatiquement transformés en hyperliens. 
L'ensemble des actions conduit à l'exécution du projet, mais il est nécessaire de souligner que l'activité linguistique chez le sujet apprenant suit, elle aussi, une progression (Figure 1) dont l'objectif est l'acquisition de points linguistiques au cours d'un processus de conceptualisation. Cette progression linguistique est sous-jacente à l'exécution du projet actionnel. En début de parcours, après avoir pris connaissance du projet à réaliser, le visionnage de vidéos ou la lecture de documents authentiques exposent l'apprenant aux faits de langues qu'il va peu à peu être amené à découvrir et approfondir. Les premières activités lui permettent de repérer les formes que l'auteur du didacticiel souhaite mettre en valeur. L'apprenant effectue tout d'abord plusieurs activités d'identification autour d'un même point linguistique. Peu à peu, ces activités font place à des activités de manipulation fondées sur le classement et la comparaison de formes linguistiques en contexte. Progressivement, des activités de production plus ou moins guidées sont introduites, et la situation, qui en est le cadre d'exécution, assure ou favorise l'emploi des formes linguistiques étudiées.

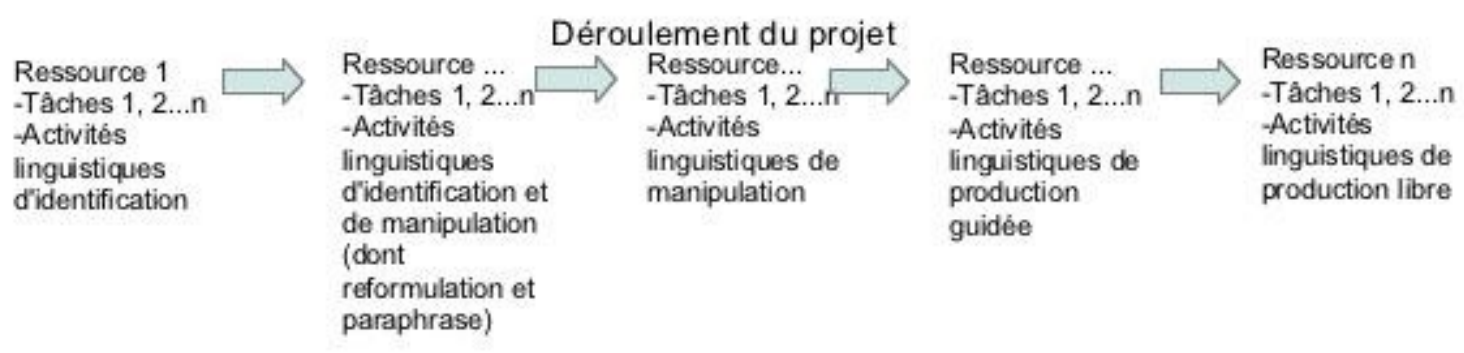

Figure 1 : Ordre didactique de la progression linguistique

en fonction de déroulement du projet proposé à l'apprenant

Une fois établi le principe organisationnel du parcours, la conception implique de prendre en compte la modalité de type distanciel et son impact sur la transmission des contenus grammaticaux. En effet, la distance imposée entre l'apprenant et l'enseignant implique une prise en charge précise et rigoureuse des étapes d'apprentissage linguistique. Par conséquent, l'ergonomie du parcours met en œuvre ces étapes, et permet à l'utilisateur apprenant d'évoluer dans le parcours actionnel.

\section{Ergonomie}

Certaines contraintes sont à prendre en compte lors de la conception d'un module sur une plate-forme :

- La contrainte distancielle implique une réflexion sur le «feedback» qui doit faire l'objet d'une attention particulière puisqu'elle est liée à chaque activité et qu'aucun présentiel ne vient compenser les éventuels manques.

- Les «affordances » des ressources mises en ligne constituent aussi une forme de contrainte, puisque l'usage qui en sera fait doit correspondre à leur raison d'être dans le parcours. Il est en effet essentiel d'identifier précisément les raisons de l'usage d'une ressource :

. s'il s'agit d'une simple lecture, un document pdf imprimable s'impose afin d'éviter les éventuels problèmes de compatibilité entre logiciels applicatifs et types de fichiers ;

. si le document à lire est destiné à être modifié (pour le faire évoluer), alors la ressource doit être éditable par un ou plusieurs individus ;

. si, lors de la lecture d'un texte, l'apprenant doit identifier des formes en cliquant sur 
certains mots, alors l'interactivité du document s'impose ;

Chaque modalité d'usage a des implications sur le choix de l'outil de création. Ces dimensions sont donc à prendre en compte, mais dans cet article, nous restreignons notre propos à la composante grammaticale.

L'ergonomie de ce parcours repose sur la construction de pages ordonnées de manière cohérente. Le parcours s'appuie sur le Content Management System (CMS) Moodle. La page d'accueil comprend des liens qui structurent le cours. Chaque lien est intuitif car il correspond à une action principale du projet comme, par exemple, "Comprendre le manuel d'utilisation d'un télescope », et ouvre la voie à un ensemble de tâches et activités de langue. Cette page d'accueil est donc le point d'entrée de plusieurs chemins actionnels qui constituent le projet. Chacun est composé d'une série de pages liées les unes aux autres, ce qui assure, en profondeur, la structuration de l'ensemble.

À chaque chemin correspondent une ou deux ressources authentiques multimédia didactisées, telles que des vidéos ou des textes. L'objectif informationnel en lien avec le projet est traité par la mise en place d'activités interactives individuelles de classement d'information. Ces activités visent à aider l'apprenant dans sa compréhension orale ou écrite des documents, et le classement d'information le place dans la démarche de construction d'une solution pour le projet. Chaque information peut être réinvestie dans une étape ultérieure du projet.

Les mêmes documents authentiques servent aussi de support pour des activités de langue. En plus des activités purement lexicales, les points linguistiques font l'objet d'un traitement particulier. Au début du parcours, après la phase d'exposition, sont d'abord mises en place des activités d'observation et d'identification de faits de langue. En milieu de parcours, et avec d'autres documents supports, des activités de comparaison, de classement, de sélection sont proposées sur le même point linguistique, qui est traité en fin de parcours lors d’activités de production guidée puis libre.

Pour illustrer notre propos, étudions l'exemple d'un module concernant les relations entre locataires et propriétaires créé dans le cadre d'un parcours de préparation à la mobilité sortante proposé à des étudiants de Licence 3 à Master 2 de l'Université de Rennes 1. Le projet global de l'apprenant est la gestion quotidienne d'un appartement avec les colocataires et le propriétaire. La tâche finale, annoncée dès l'introduction, est de participer à une discussion entre colocataires pour se mettre d'accord sur des règles de vie. Le module observé correspond aux demandes faites au propriétaire en cas de problème.

La première tâche correspond à la compréhension des informations. L'apprenant doit progressivement reconstruire un dialogue en choisissant l'extrait audio qui correspond à la suite de l'échange. L'apprenant doit donc se concentrer sur la pertinence des informations. La vidéo d'introduction sert de déclencheur en proposant le contexte de départ. Trois autres extraits vidéos constituent trois réponses possibles, mais une seule correspond à la suite effective de la conversation. L'apprenant doit donc faire correspondre des contextes informationnels.

Une fois l'information globale maîtrisée par l'apprenant, les détails sont abordés. L'apprenant travaille à la fois sa compétence de compréhension par discrimination auditive et identifie les structures syntaxiques propres au questionnement poli, ultérieurement utile pour produire un document écrit adressé à un propriétaire (landlord). La figure 2 présente une activité de discrimination auditive placée après les activités de compréhension de l'information générale. Il s'agit d'identifier les phonèmes pour reconstituer la chaîne de morphèmes qui, du point de vue fonctionnel, sert à exprimer une demande polie. Grâce aux 
activités précédentes, l'apprenant a compris les informations demandées poliment. Ici, il s'arrête sur la manière dont sont formulées ces demandes en liant la réalisation fonctionnelle de la demande polie à sa réalisation syntaxique.

\section{How did the tenant ask her landlord for help?}

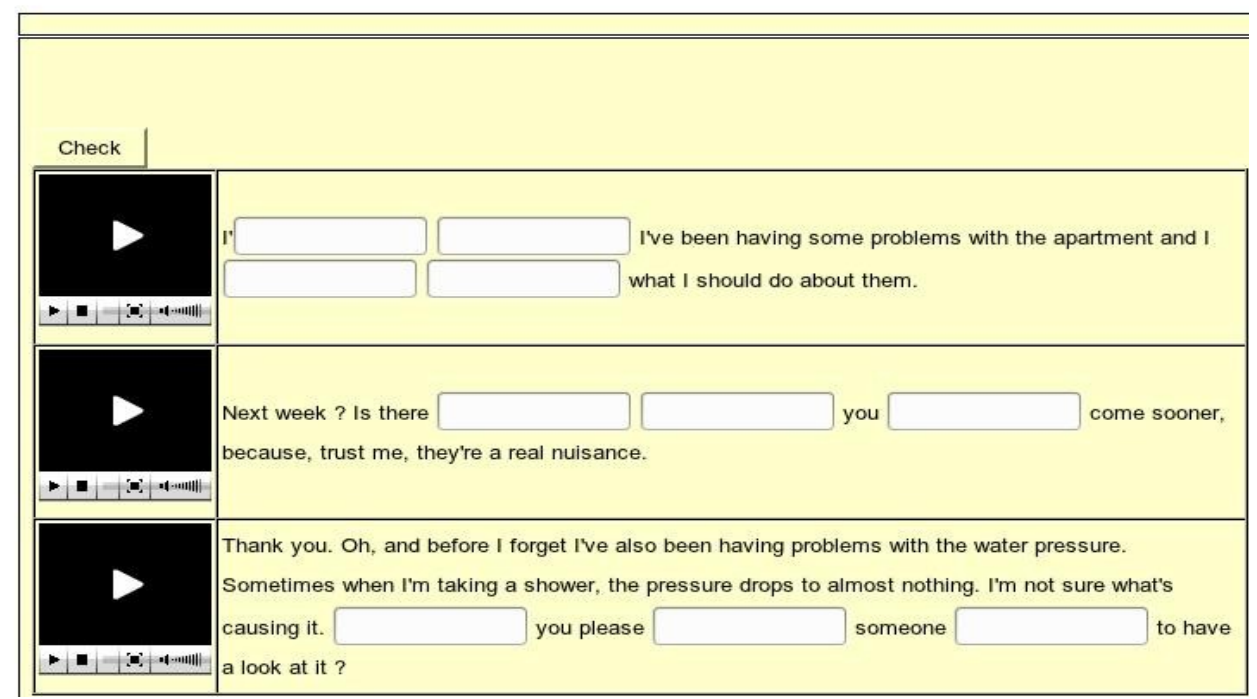

Figure 2 : Compréhension détaillée des questions polies en contexte

L'activité de manipulation de la figure 3 ci-dessous reprend le contexte et incite l'apprenant à identifier les questions en mode audio, tout en les classant en fonction de leur pertinence au vu des descripteurs thématiques présents dans la colonne de gauche : il y a un va-et-vient entre forme linguistique et information en contexte.

\section{Asking for help.}

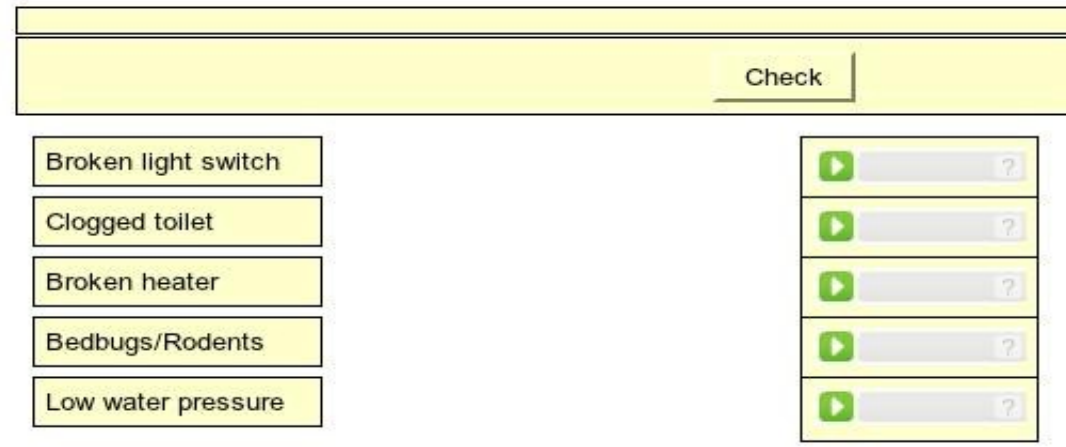

Figure 3 : Identification des questions en contexte 
Une fois les formes linguistiques repérées et entendues en contexte, leur manipulation aborde la question de l'ordre syntaxique, qui génère une réflexion sur les règles régissant l'agencement des mots. Des activités de recomposition d'un énoncé - dont le contexte est illustré par des photos - sont proposées. Les mots de chaque énoncé doivent être remis dans l'ordre. Chaque photo a pour but de proposer un contexte suffisamment précis pour déclencher un certain type de question : par exemple, une demande d'aide pour se débarrasser d'une souris dans le cas du module sur le logement. La photo déclenche le besoin fonctionnel et l'apprenant met en œuvre la réalisation syntaxique. Cependant, il n'a pas encore à produire librement son énoncé puisque les mots sont imposés. À cette étape, est ainsi générée une réflexion sur les règles syntaxiques de la question et de la modalité pour reconstruire une demande polie.

L'activité de recomposition d'énoncé cède la place à une autre forme de production guidée (Figure 4) où l'objectif fonctionnel reste le même (demande polie). L'ordre des choix effectués par l'utilisateur suit l'ordre diachronique de la production. L'apprenant lit de manière active en faisant des choix contextuels et syntaxiques.

\section{Polite Requests 4}

Bulld the sentence by choosing one of the given options. If your choice is correct, the sentence will be added to the sentence.

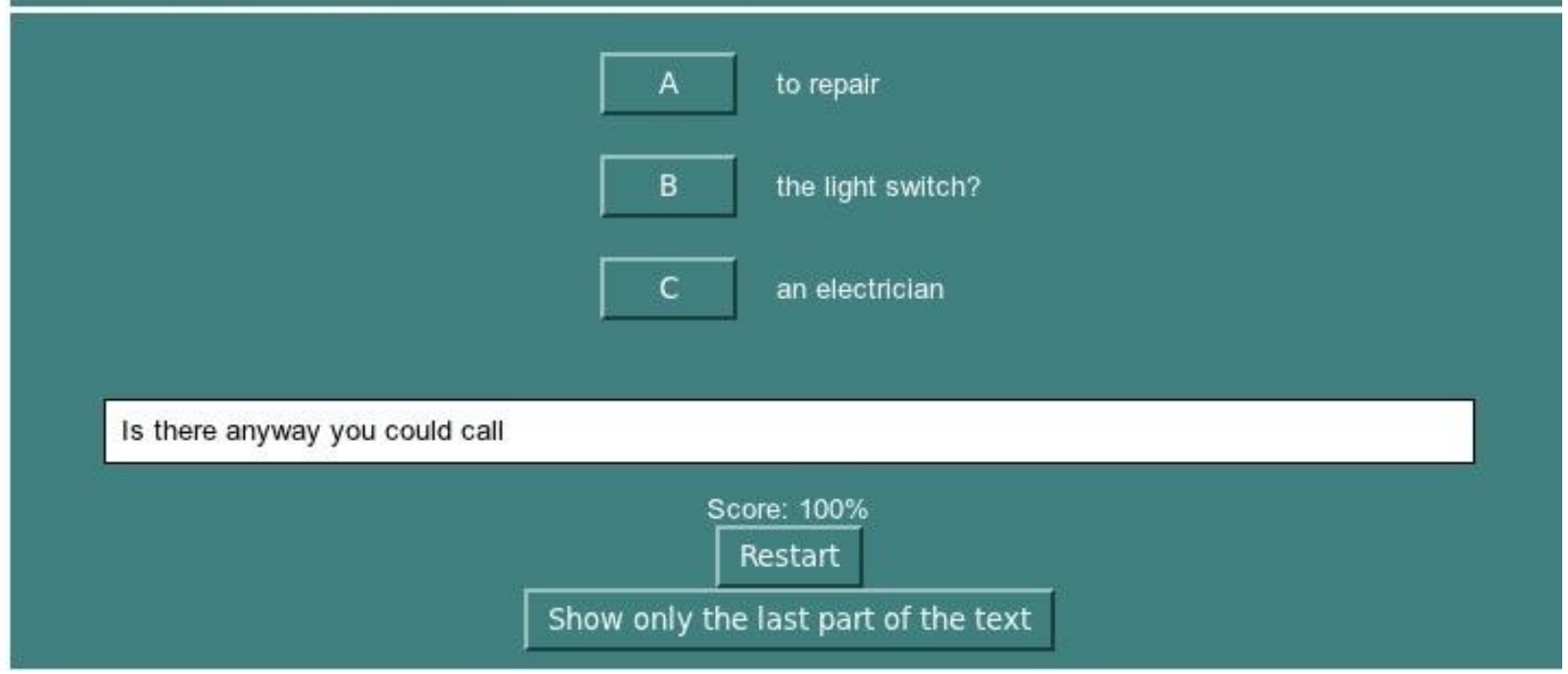

Figure 4 : Production guidée de questions

Tout au long de ses activités, l'apprenant se trouve constamment exposé aux mêmes structures syntaxiques et à leur réalisation dans des contextes différents et au sein d'activités différentes dont les feedbacks sont différents. Le repérage, l'identification puis la production d'énoncés commençant, par exemple, par " Would you mind + ING » ou " I was wondering if + S + COULD », favorisent l'identification de ce modèle de question aux types de situation dans lesquelles elles sont posées. Les valeurs dominantes de l'hypothétique portées par «-ED» associé à la visée (WILL) ou à la capacité (CAN) émergent des énoncés contextualisés, des types d'activités et de leurs feedbacks. Par ce biais, l'apprenant émet, seul, des hypothèses sur l'utilisation de ces formules de politesse avec des interlocuteurs ayant un statut particulier marqué par la distance vis-à-vis de l'énonciateur. La nécessité d'introduire cette rupture par l'usage du 
prétérit devient le point commun de multiples énoncés. Progressivement, l'apprenant est amené à conceptualiser l'usage de cet objet syntaxique qu'est la demande polie. Reste la phase de transfert dans un contexte nouveau. La dernière phase du parcours peut, selon le module, être réalisée à l'écrit ou à l'oral avec un tuteur. Dans l'exemple étudié, l'apprenant bénéficie d'une rencontre avec un tuteur pour participer à un jeu de rôle dont l'objectif lui est décrit en ces termes :

Now, to complete your final task, make an appointment with a course tutor at the resource centre.

During your meeting with this tutor, you will enact a role-play. The two of you will be roommates who have recently moved in together. In order to avoid conflict, you would like to agree on some basic ground rules. To help you with this task, print out this roommate agreement form. Make sure you discuss each section of the agreement.

La situation imposée par le jeu de rôle implique, entre autres, l'usage de « -ED » de rupture pour atténuer la valeur d'assertion si les demandes étaient faites au temps grammatical présent. En d'autres termes, la situation suscite l'usage de «-ED » afin de respecter la norme de politesse propre à ce type d'échanges. Cette démarche progressive mène donc de la simple identification de formes à la conceptualisation d'un constituant implicite.

\section{Conclusion}

Cet article traite des étapes de construction d'un didacticiel d'apprentissage de l'anglais et démontre, à propos du scénario d’apprentissage, la nécessité de mener de front deux types de déroulement :

- un déroulement pédagogique dans lequel l'apprenant puise le sens de son travail. Il se voit proposer un projet décliné en un certain nombre d'actions impliquant des activités de compréhension et d'expression. Cette perspective actionnelle sert de catalyseur pour l'implication de l'apprenant ;

- un déroulement linguistique assurant l'assimilation de compétences linguistiques dont l'expérience en classe montre qu'elles sont mal acquises ou maîtrisées par les apprenants au niveau L2.

Cette dualité engendre une approche particulière de conception. L'approche exposée ici défend le fait que le processus d'élaboration d'un parcours peut résulter de la confrontation de la démarche actionnelle portée par le CECRL, et de la pratique raisonnée de la langue portée par les Instructions Officielles des années 80.

Cette confrontation ne se solde pas par la subordination de l'une à l'autre (Chini 2009), mais plutôt par leur agencement mutuel. Nous considérons en effet que la démarche actionnelle devrait appeler la pratique raisonnée de la langue par le biais de phases d'élucidation ou d'élaboration du sens par les apprenants. Si le travail de raisonnement / réflexion sur les formes linguistiques, et les opérations qui les sous-tendent, se trouve subordonné au travail actionnel, il demeure, dans les faits, l'objectif principal pour l'auteur du didacticiel, enseignant d'anglais. L'action est un prétexte, la composante linguistique représentant son cœur de métier. Pour l'apprenant, l'action peut être source de motivation, et le travail sur la langue intervient en fonction de ses besoins au fur et mesure qu'ils apparaissent en contexte. Ceci offre à l'apprenant une réflexion sur la langue en contexte, et l'accession à la perception des « enjeux langagiers » (Chini 2009).

L'action conceptualisante de l'apprenant devrait être au centre du processus d'apprentissage. La compréhension et la maîtrise des opérations sous-jacentes au fonctionnement linguistique devraient être 
des objectifs constamment présents au sein d'un parcours d'apprentissage. Ces objectifs ne peuvent, cependant, se suffire à eux-mêmes au risque de transformer le parcours en une leçon de grammaire en ligne. Le parcours, qui représente un projet actionnel pour l'apprenant, distille l'analyse de points linguistiques lors du déroulement des actions, et incite à la conceptualisation. L'action soutient la conceptualisation et la motive. Au final, cette démarche tente de rendre l'apprenant autonome du point de vue de son apprentissage linguistique. Elle s'inscrit dans un dispositif d'Auto-formation Guidée (AFG) qui peut représenter une solution viable face aux contraintes structurelles qui pèsent sur l'enseignement des langues en LANSAD.

Bibliographie

Barbazan, M. 2011. «Intégrer les Tice à une approche cognitive de la grammaire du discours ». Alsic, Vol. 14. <http://alsic.revues.org/2337> (consulté le 27 juin 2012).

Bulletin officiel. 2010. « Enseignement commun LV1 et LV2 : du niveau A2 vers le niveau B1 ». Bulletin officiel Spécial $n^{\circ} 4$. Ministère de l’Éducation Nationale.

Chini, D. 2009. «Linguistique et didactique : où en est-on? Quelle place pour une approche conceptualisante de la construction de la langue dans la perspective actionnelle ? ». Les Cahiers de l'Acedle, $n^{\circ} 6$ (2) : 129-153.

Conseil de l'Europe. 2001. Cadre européen commun de référence pour les langues : apprendre, enseigner, évaluer. Paris : Les Éditions Didier.

Ellis, R. 2003. Task-based language learning and teaching. Oxford : Oxford University Press.

Frei, H. 2011 [1929]. La grammaire des fautes. Vol. 1 Genève : Suisse.

Ministère de l’Éducation nationale, de la recherche et de la technologie. 1998. Anglais classes de Seconde, Première et Terminale. Paris : Centre national de documentation pédagogique.

Portine, H. 2009. «La linguistique substrat du discours didactique : quand l'histoire nous parle au présent ». Les Cahiers de l'Acedle, $n^{\circ} 6$ (2) : 13-37.

Uyttebrouck, E. 2005. « Mais pourquoi diable ne lisent-ils pas mes notes de cours en ligne ? ». Journal of Distance Education, 20 (1) : 39-59.

Thomas Gaillat, en poste à l'Université de Rennes 1, est enseignant d'anglais depuis 1999 ; il est membre de CLILLAC ARP et LIDILE. Il prépare une thèse à l’Université Paris-Diderot au sujet de l'interopérabilité des corpus d'apprenants en anglais ; ses recherches portent plus particulièrement sur l'annotation des démonstratifs this et that.

Ses publications portent sur des problématiques de linguistique anglaise liées aux domaines du traitement automatique des langues et de l'acquisition d'une langue étrangère.

thomas.gaillat@univ-rennes1.fr

Martine Schuwer est professeur de linguistique anglaise à l'Université Rennes 2 et membre de LIDILE, EA 3874. 
martine.schuwer@gmail.com

Sophie Belan est maître de conférences en anglais à l'Université de Nantes et membre de CRINI EA 1162 et LIDILE EA 3874. Ses recherches et publications portent sur l'utilisation des TICE pour l'enseignement/apprentissage des langues, et plus particulièrement sur les dispositifs hybrides d'enseignement/apprentissage de l'anglais.

sophie.belan@univ-nantes.fr 